\title{
DEMENTIA INPATIENT THERAPEUTIC INTERVENTIONS IN A SCOTTISH HEALTH REGION
}

\author{
Gary Stevenson, Stuart Semple \& Hinesh Topiwala \\ Department of Psychiatry, NHS Fife, Fife, Scotland KY15 5RR (gstevenson@nhs.net)
}

\section{Background \& Objectives}

- There is published evidence of the benefits and limitations, including potential harm, of psychotropic medication and benefits of nonpharmacological interventions for the management of challenging behaviours associated with dementia (BPSD).

- To examine the usage of such interventions in the management of BPSD within $3 \mathrm{NHS}$ 'specialist' dementia inpatient wards (56 beds) in a Scottish health region (Fife, population 365k) and to identify targets for service improvements.

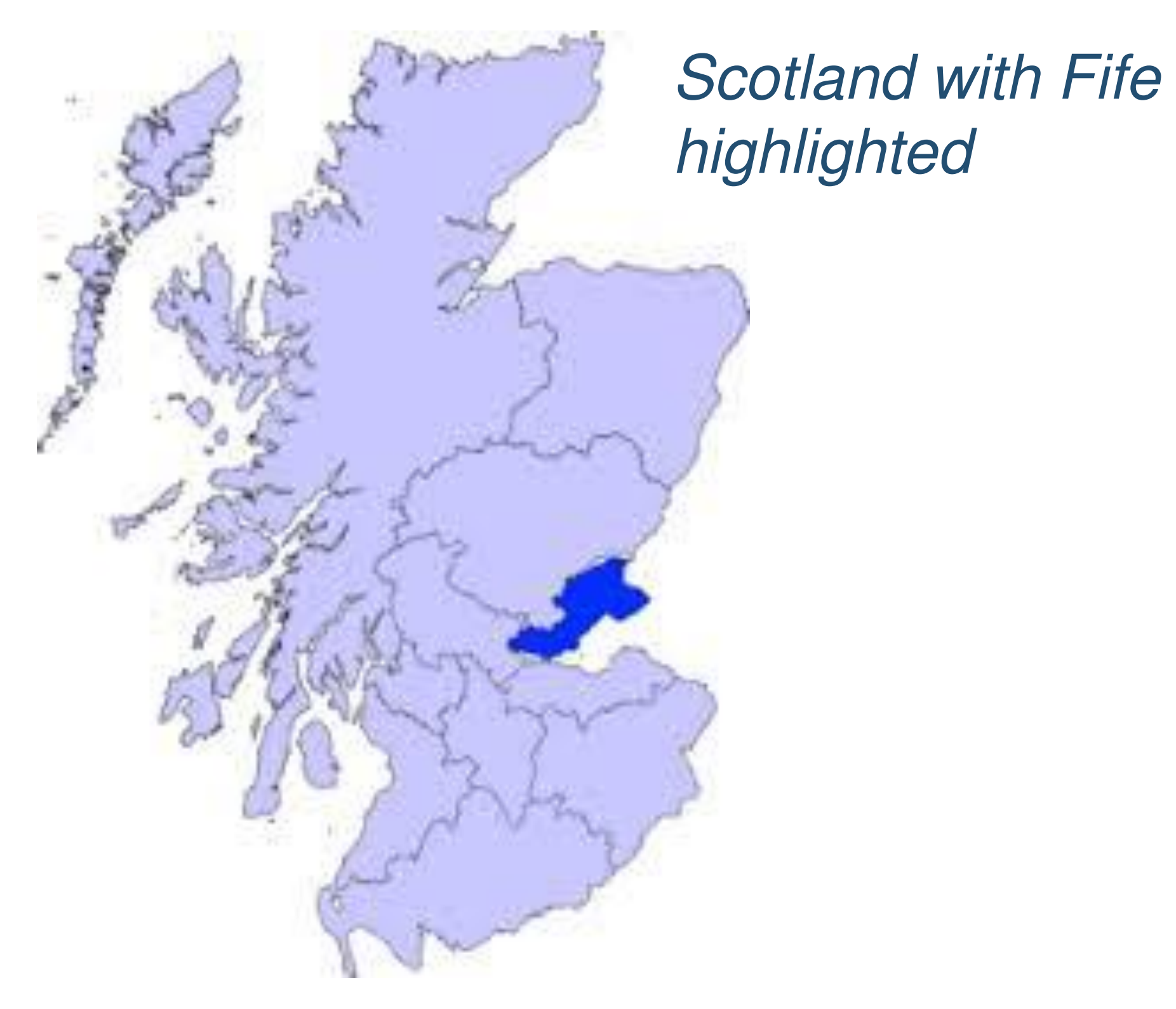

\section{Methods}

Patient demographic data, diagnoses, psychotropic prescriptions, multidisciplinary team input, and evidence of individualised non-pharmacological interventions for BPSD were collected in January 2018 from patient notes, care plans and medication charts.

\section{Results}

- Identified 50 older patients with dementia who had spent on average 15 months within hospital.

- Most had multiple medical co-morbidities.

- All lacked capacity to consent to their general care and medical treatments.

- $26 \%$ were detained in hospital under Scottish Mental Health legislation.

- 56\% were prescribed an antipsychotic (Risperidone 16\%), 36\% a cognitive enhancer (Memantine or a cholinesterase inhibitor), 58\% an antidepressant (Trazodone $26 \%$ ), and $90 \%$ a benzodiazepine.

- There was limited regular multi-professional team working identified in the wards.

- Of the non-pharmacological therapeutic interventions, 38\% were receiving music therapy, $14 \%$ massage, $14 \%$ multisensory inputs, 8\% Playlist for Life (an individualised music profile), 2\% Art therapy and $2 \%$ Pet therapy.

\section{Conclusions}

- For this inpatient population with BPSD there were high rates of offlicence drug prescribing with limited investment in evidence-based non-pharmacological therapeutic interventions which may offer alternatives to pharmacological requirements.

- The Scottish Government publication (June 2018) of "Transforming Specialist Dementia Hospital Care" is to be welcomed in raising the profile of this "overlooked" inpatient population.

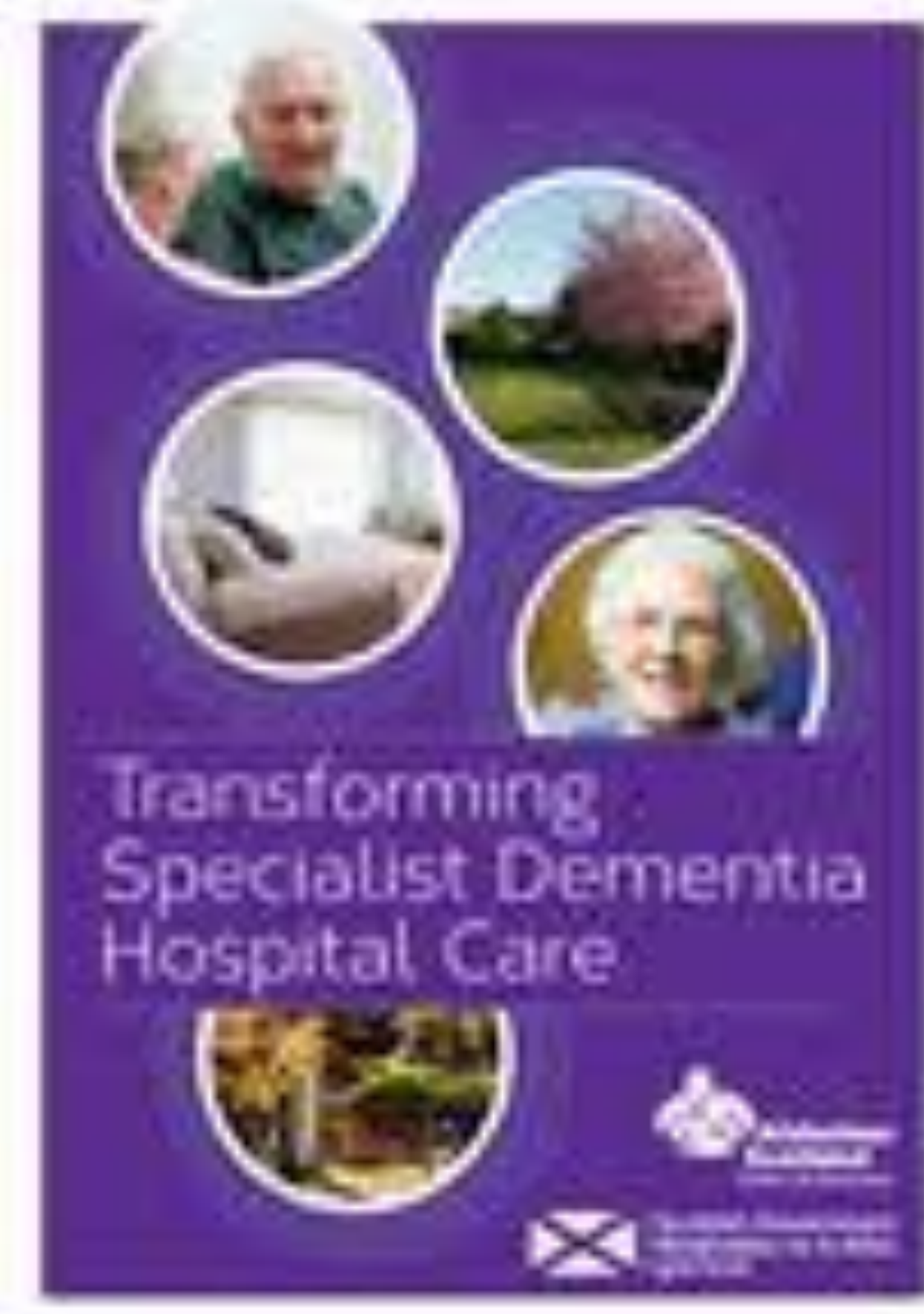

